\title{
Prevalence of thyroid nodules and their associated clinical parameters: a large-scale, multicenter-based health checkup study
}

Jae Hoon Moon ${ }^{1,}$, Min Kyung Hyun ${ }^{2, *}$, Ja Youn Lee ${ }^{3}$, Jung Im Shim³, Tae Hyuk Kim ${ }^{4}$, Hoon Sung Choi ${ }^{5}$, Hwa Young Ahn ${ }^{6}$, Kyung Won Kim ${ }^{7}$, Do Joon Park ${ }^{4}$, Young Joo Park ${ }^{3,4}$, and Ka Hee Yi ${ }^{8}$

${ }^{1}$ Department of Internal Medicine, Seoul National University Bundang Hospital, Seongnam; ${ }^{2}$ Department of Preventive Medicine, Dongguk University College of Korean Medicine, Gyeongju; ${ }^{3}$ National Evidence-based Healthcare Collaborating Agency, Seoul; ${ }^{4}$ Department of Internal Medicine, Seoul National University Hospital, Seoul; ${ }^{5}$ Department of Internal Medicine, Kangwon National University Hospital, Chuncheon; ${ }^{6}$ Department of Internal Medicine, Chung-Ang University Hospital, Seoul; ${ }^{7}$ Department of Internal Medicine, Seoul National University Hospital Healthcare System Gangnam Center, Seoul; ${ }^{8}$ Department of Internal Medicine, Seoul Metropolitan Government Seoul National University Boramae Medical Center, Seoul, Korea

Received: August 12, 2015

Revised : February 3, 2016

Accepted: June 13, 2016

\section{Correspondence to}

Ka Hee Yi, M.D.

Department of Internal Medicine, Seoul Metropolitan Government Seoul National University Boramae Medical Center, 20 Boramae-ro 5-gil, Dongjak-gu, Seoul 07061, Korea

Tel: +82-2-870-3203

Fax: +82-2-831-2826

E-mail: khyi@snu.ac.kr

*These authors contributed equally to this work.

Background/Aims: We evaluated the prevalence and characteristics of thyroid nodules detected by thyroid ultrasound (US) at health checkups and the associated clinical parameters.

Methods: A total of 72,319 subjects who underwent thyroid US at three health checkup centers in Korea from January 2004 to December 2010 were included in this study. The correlations between the presence of thyroid nodules and other clinical parameters were analyzed.

Results: The prevalence of thyroid nodules and cysts was 34.2\% $(n=24,757)$. Thyroid nodules were more prevalent in women and older age groups. Among the subjects with thyroid nodules with size information ( $\mathrm{n}=24,686), 18,833(76.3 \%)$ had nodules measuring $\leq 1.0 \mathrm{~cm}$. Women and older age groups showed higher proportion of larger nodules. Percentage of women, age, body mass index (BMI), waist circumference, body fat composition, blood pressure, and the level of fasting glucose, total cholesterol, and low density lipoprotein cholesterol were higher in the subjects with thyroid nodules compared to those without nodules. The prevalence of metabolic syndrome and overt/subclinical thyrotoxic state was higher in the subjects with thyroid nodules. In the multivariable logistic regression analysis, women, age, BMI, metabolic syndrome, and thyrotoxicosis were independently associated with the presence of thyroid nodules.

Conclusions: The high prevalence of thyroid nodules in people who underwent thyroid US at a health checkup suggests that increased detection of thyroid nodules resulted in an increased prevalence in the general population. However, metabolic disturbances may also have contributed to the increase in thyroid nodule prevalence in Korea.

Keywords: Thyroid nodule; Ultrasonography; Prevalence; Metabolic syndrome

\section{INTRODUCTION}

Thyroid nodules are diagnosed increasingly in clinical practice. The increasing detection rate of small and non-symptomatic thyroid nodules by various imaging methods has resulted in a high prevalence of thyroid nodules among 
the general population $[1,2]$. A previous study reported that higher levels of health-care access are associated with a higher incidence of thyroid nodules and cancers [3]. In Korea, the level of health-care access is very high; for example, $>60 \%$ of the population has undergone a health checkup in the preceding 2 years [4]. The prevalence of thyroid nodules has increased rapidly in Korea. Previous single-center studies in the mid-2000 reported a prevalence rates of $28.3 \%$ to $42.4 \%$ and $14.1 \%$ to $29.1 \%$ in asymptomatic women and men, respectively [5-8]. Despite the high probability that this is a health checkup-driven increase in the detection of thyroid pathology, large-scale and multicenter-based studies of the prevalence and characteristics of thyroid nodules detected by thyroid sonography at health checkups are lacking in Korea.

The contribution of the increasing detection rate is a widely accepted explanation for the increasing prevalence of thyroid nodules. It is well established that thyroid nodule prevalence depends on factors such as the detection method, sex, age, iodine intake, and radiation exposure [9]. Other risk factors including metabolic parameters have received attention recently. Previous studies have shown that insulin resistance was associated with increased thyroid nodule prevalence in iodine-deficient and -sufficient areas [10,11]. A recent study in China also reported that hypertension, prediabetes, and diabetes were independent risk factors for thyroid nodules [12]. In Korea, body size including height, weight, and body mass index (BMI) were reported to be associated with the presence of thyroid nodules in women [13].

In this study, we conducted a large-scale, multicenter-based study of 72,319 subjects who underwent thyroid ultrasound (US) at health checkups to investigate the prevalence and characteristics of thyroid nodules and their associated clinical parameters in the healthy Korean population.

\section{METHODS}

\section{Subjects}

Data were collected from three health checkup centers; Seoul National University Hospital Healthcare System Gangnam Center (SNUH-HSGC, Seoul, Korea), Seoul National University Bundang Hospital Health Promotion Center (SNUBH-HPC, Seongnam, Korea), and
Seoul Metropolitan Government Seoul National University Boramae Medical Center Health Promotion Center (SNUBMC-HPC, Seoul, Korea). A total of 254,222 health checkups were performed from January 2004 to December 2010. Among these cases, thyroid US was performed in 100,971 cases. After excluding checkups of the 495 subjects who had undergone thyroid surgery and detecting repeated checkups, a total of 72,319 subjects were included in this study. In case of repeated checkups, data of the first checkup were used in the analyses. We did not exclude the subjects with other medical history, including diabetes, hypertension, dyslipidemia, and thyroid dysfunction as well as the subjects using any kind of medication, including thyroid hormone or antithyroid drugs. Informed consent was obtained. This study was approved by the Institutional Review Boards of the SNUH (H-1106-113-367), SNUBH (B-1303/194-109), and SNUBMC (26-2013-15/042).

\section{Anthropometric parameters}

We measured the height and weight of subjects in light clothing and without shoes to the nearest $0.1 \mathrm{~cm}$ and 0.1 $\mathrm{kg}$, respectively. BMI was calculated as the ratio between weight and the square of height, and was expressed in $\mathrm{kg} / \mathrm{m}^{2}$. Waist circumference was measured at the midpoint between the lateral iliac crest and the lowest rib. Total body fat percentage was determined using bioelectrical impedance analysis (Inbody 720, Biospace, Seoul, Korea). A standard mercury sphygmomanometer was used to measure systolic blood pressure (SBP) and diastolic blood pressure.

\section{Biochemical and hormonal parameters}

Plasma glucose concentration was measured in the 12-hour-fasted state using the glucose oxidase method (Hitachi 747 chemistry analyzer, Hitachi, Tokyo, Japan). Glycosylated hemoglobin (HbAic) level was measured using a Bio-Rad Variant II Turbo HPLC analyzer (Bio-Rad, Hercules, CA, USA). The serum concentrations of aspartate aminotransferase (AST), alanine aminotransferase (ALT), $\gamma$-glutamyl transpeptidase (GGT), blood urea nitrogen (BUN), creatinine, uric acid, total cholesterol, triglycerides, low density lipoprotein cholesterol (LDL-C), and high density lipoprotein cholesterol (HDL-C) were measured with a Hitachi 747 chemistry analyzer. The concentrations of serum free thyroxine $\left(\mathrm{T}_{4}\right)$ and thyroid 
stimulating hormone (TSH) were measured using immunoradiometric assays (free $\mathrm{T}_{4}$, DiaSorin S.p.A., Saluggia, Italy; TSH, CIS bio international, Gif-sur-Yvette, France). The reference ranges were 0.7 to $1.8 \mathrm{ng} / \mathrm{dL}$ and 0.4 to $4.1 \mathrm{mIU} / \mathrm{L}$ for free $\mathrm{T}_{4}$ and $\mathrm{TSH}$, respectively.

\section{Classification of thyroid function status}

The subjects were divided in five categories based on their thyroid function status: (1) overt hypothyroid state was defined as a TSH level $>4.10 \mathrm{mIU} / \mathrm{L}$ with a free $\mathrm{T}_{4}$ level < $0.7 \mathrm{ng} / \mathrm{dL}$; (2) subclinical hypothyroid state was defined as a TSH level $>4.10 \mathrm{mIU} / \mathrm{L}$ with a normal free T4 level ( 0.7 to $1.8 \mathrm{ng} / \mathrm{dL}$ ); (3) euthyroid state was defined as a normal TSH level (0.40 to $4.10 \mathrm{mIU} / \mathrm{L})$; (4) subclinical thyrotoxic state was defined as a TSH level $<0.40$ $\mathrm{mIU} / \mathrm{L}$ with a normal free $\mathrm{T}_{4}$ level; and (5) overt thyrotoxic state was defined as a TSH level $<0.40 \mathrm{mIU} / \mathrm{L}$ with a free $\mathrm{T}_{4}$ level $>1.8 \mathrm{ng} / \mathrm{dL}$. We did not consider the use of thyroid hormone and antithyroid drugs in defining thyroid function status.

\section{Diagnosis of metabolic syndrome}

According to guidelines from the National Heart, Lung, and Blood Institute and the American Heart Association [14], metabolic syndrome (MetS) is diagnosed when a patient has at least three of the following five conditions: (1) fasting glucose $\geq 100 \mathrm{mg} / \mathrm{dL}$ or receiving drug therapy for hyperglycemia; (2) blood pressure $\geq 130 / 85$ $\mathrm{mmHg}$ or receiving drug therapy for hypertension; (3) triglycerides $\geq 150 \mathrm{mg} / \mathrm{dL}$ or receiving drug therapy for hypertriglyceridemia; (4) HDL-C < $40 \mathrm{mg} / \mathrm{dL}$ in men or $<50 \mathrm{mg} / \mathrm{dL}$ in women or receiving drug therapy for reduced HDL-C; and (5) waist circumference $\geq 90 \mathrm{~cm}$ in men or $\geq 80 \mathrm{~cm}$ in women.

\section{Thyroid ultrasound and the classification of thyroid nodules}

All ultrasonographic examinations on the thyroid were performed in each health checkup center by several radiologists using high-resolution US equipped with 7.5 to 12 or 8 to $15 \mathrm{MHz}$ linear transducers. Thyroid nodules were defined as one or more discrete lesions that were within the thyroid gland but were radiologically distinct from the surrounding thyroid parenchyma. The length, width, and depth of each nodule were measured at their longest aspect on the US images, which were saved as
Digital Imaging and Communications in Medicine images. All US reports were also saved as digital text. We extracted data of the presence and size of nodules from the free text of the thyroid US reports using a computerized keyword extraction method (Java Development Kit version 1.7, Oracle Corp., Redwood Shores, CA, USA). All extracted data from 72,319 cases of thyroid US report were reviewed by a medical record administrator and he fixed typo errors and corrected the data according to the standardized input format. These preliminarily reviewed data were re-reviewed by two physicians (H.Y.A. and K.W.K.). These physicians reevaluated the digital images of 10,594 cases (H.Y.A., 5,231 cases; K.W.K., 5,363 cases) harboring the keyword "cyst" or "colloid" without "nodule" and assigned them to one of two categories. According to the lesion's content, a lesion in which the cystic portion comprised $\geq 95 \%$ of the volume was defined as a pure cyst, and a lesion in which the solid portion comprised $6 \%$ to $100 \%$ of the volume was defined as a nodule. If the extracted data harbored keywords "cyst" and "nodule" in one subject, this was classified as a nodule. Seventy-one subjects whose extracted data harboring "nodule" without size information were excluded in the analyses including size information. In the subjects with multiple nodules, the size of the largest thyroid nodule was used in the analyses.

\section{Statistical analyses}

Values with normal distribution were expressed as mean $\pm \mathrm{SD}$, and values with nonnormal distribution were expressed as median (interquartile range). The anthropometric and metabolic parameters were compared between the subjects with and without thyroid nodules using Student $t$ test, chi-square test, or a linear-by-linear association test. Multivariable logistic regression analysis was used to estimate multiple correlations between the presence of thyroid nodules and other factors. All statistical analyses were performed using SAS statistical software version 9.4 (SAS Institute, Cary, NC, USA) and Stata/MP version 14 (StataCorp LP, College Station, Texas, USA). Data with a $p<0.05$ were considered significant.

\section{RESULTS}

Prevalence of thyroid nodules and pure cysts in subjects 
Table 1. The prevalence of thyroid nodules according to age and sex

\begin{tabular}{|c|c|c|c|c|c|c|c|}
\hline \multirow[b]{2}{*}{ Variable } & \multicolumn{2}{|c|}{ Total subject } & \multicolumn{2}{|c|}{ Men } & \multicolumn{2}{|c|}{ Women } & \multirow[b]{2}{*}{$p$ value ${ }^{a}$} \\
\hline & $\begin{array}{c}\text { Subjects } \\
\text { underwent } \\
\text { thyroid US }\end{array}$ & $\begin{array}{c}\text { Subjects with } \\
\text { thyroid } \\
\text { nodules }\end{array}$ & $\begin{array}{c}\text { Subjects } \\
\text { underwent } \\
\text { thyroid US }\end{array}$ & $\begin{array}{c}\text { Subjects with } \\
\text { thyroid } \\
\text { nodules }\end{array}$ & $\begin{array}{c}\text { Subjects } \\
\text { underwent } \\
\text { thyroid US }\end{array}$ & $\begin{array}{c}\text { Subjects with } \\
\text { thyroid } \\
\text { nodules }\end{array}$ & \\
\hline Total & 72,319 & $24,757(34.2)$ & 36,710 & $9,919(27.0)$ & 35,609 & $14,838(41.7)$ & $<0.001$ \\
\hline \multicolumn{8}{|c|}{ Age group, yr } \\
\hline$<30$ & 1,935 & $250(12.9)$ & 718 & $55(7 \cdot 7)$ & 1,217 & $195(16.0)$ & 0.001 \\
\hline $30-39$ & 11,411 & $2,378(20.8)$ & 5,211 & $777(14.9)$ & 6,200 & $1,601(25.8)$ & $<0.001$ \\
\hline $40-49$ & 23,649 & $6,933(29 \cdot 3)$ & 12,213 & 2,671 (21.9) & 11,436 & $4,262(37 \cdot 3)$ & $<0.001$ \\
\hline $50-59$ & 22,113 & $8,655(39 \cdot 1)$ & 11,467 & $3,547(30.9)$ & 10,646 & $5,108(48.0)$ & $<0.001$ \\
\hline $60-69$ & 10,763 & $5,197(48.3)$ & 5,738 & $2,259(39.4)$ & 5,025 & $2,938(58.5)$ & $<0.001$ \\
\hline$\geq 70$ & 2,448 & $1,344(54 \cdot 9)$ & 1,363 & $610(44.8)$ & 1,085 & $734(67 \cdot 6)$ & $<0.001$ \\
\hline$p$ value ${ }^{b}$ & \multicolumn{2}{|c|}{$<0.001$} & \multicolumn{2}{|c|}{$<0.001$} & \multicolumn{2}{|c|}{$<0.001$} & - \\
\hline
\end{tabular}

Values are presented as number (\%).

US, ultrasound.

${ }^{a}$ Derived from the chi-square test between sex and the presence of thyroid nodules.

${ }^{\mathrm{b}}$ Derived from a linear-by-linear association test between age groups and the presence of thyroid nodules.

who underwent thyroid ultrasound at health checkups A total of 72,319 subjects (men:women, 36,710:35,609) who underwent thyroid US in their health checkups were included in this study. Mean age of included subjects was $49.5 \pm 10.3$ years and mean BMI was $23.5 \pm 3.0 \mathrm{~kg} / \mathrm{m}^{2}$. Thyroid nodules were found in 24,757 subjects $(34.2 \%)$ and 9,060 subjects (12.5\%) had only pure cysts. Among the 36,710 men, 9,919 subjects (27.0\%) and 4,169 (11.4\%) were diagnosed with thyroid nodules and pure cysts, respectively. Among the 35,609 women, 14,838 subjects (41.7\%) and 4,891 (13.7\%) were diagnosed with nodules and pure cysts, respectively. The thyroid nodule prevalence increased with age in both men and women (Table 1) but the prevalence of pure cysts did not differ according to age (data not shown). In all subjects and in each age group, thyroid nodule prevalence was higher in women than in men (Table 1). The thyroid nodule prevalence per year in those who underwent thyroid US did not change from $2004(32.9 \%)$ to 2010 (36.1\%).

\section{Size distribution of thyroid nodules}

Among the 24,686 subjects diagnosed as thyroid nodules with specific size information, 18,833 (76.3\%) had nodules measuring $\leq 1.0 \mathrm{~cm}$ (Table 2). Women and older age groups showed a higher proportion of nodules measuring $>1.0 \mathrm{~cm}$ (Table 2).

\section{Comparison of anthropometric and metabolic param- eters according to the presence of thyroid nodules}

After excluding 9,060 subjects who had only pure cysts, we divided the remaining 63,188 subjects into two groups: those without thyroid nodule (non-nodular group) and those with thyroid nodules (nodular group). Between these two groups, subjects with thyroid nodules were older and showed higher percentage of women than those without thyroid nodule (Table 3). BMI, waist circumference, body fat composition, blood pressure, and the level of fasting glucose, HbArc, BUN, total cholesterol, HDL-C, and LDL-C were higher in the nodular group in compared with the non-nodular group (Table 3). The concentrations of AST, ALT, GGT, creatinine, uric acid, triglycerides, and TSH were lower in the nodular groups in compared with non-nodular group (Table 3). The prevalence of MetS was higher in the nodular group and the subjects in the nodular group had higher number of components satisfying MetS criteria (Table 3). The nodular group showed a higher frequency of overt/subclinical thyrotoxic state and a lower frequency of overt/subclinical hypothyroid state (Table 3).

\section{Clinical parameters associated with the presence of thyroid nodules}

In the comparison of anthropometric and metabolic parameters according to the presence and size of thy- 
Table 2. Size-distribution of thyroid nodules with the longest diameter

\begin{tabular}{|c|c|c|c|c|c|c|}
\hline \multirow{2}{*}{ Variable } & \multirow{2}{*}{ Total no. } & \multicolumn{4}{|c|}{ Size, $\mathrm{cm}$} & \multirow{2}{*}{$p$ value } \\
\hline & & $\leq 0.5$ & $0.5-1.0$ & $1.0-2.0$ & $>2.0$ & \\
\hline Total subjects with thyroid nodules & 24,686 & $9,062(36.7)$ & $9,771(39.6)$ & $4,604(18.7)$ & $1,249(5.0)$ & \\
\hline Sex & & & & & & $<0.001$ \\
\hline Men & 9,900 & $3,813(38.5)$ & $3,942(39.8)$ & $1,701(17.2)$ & $444(4 \cdot 5)$ & \\
\hline Women & 14,786 & $5,249(35 \cdot 5)$ & $5,829(39.4)$ & $2,903(19.6)$ & $805(5 \cdot 5)$ & \\
\hline Age group, yr & & & & & & $<0.001$ \\
\hline$<30$ & 247 & $99(40.1)$ & $90(36.4)$ & $45(18.2)$ & $13(5 \cdot 3)$ & \\
\hline $30-39$ & 2,369 & $994(42.1)$ & $903(38.1)$ & $368(15 \cdot 5)$ & $104(4 \cdot 3)$ & \\
\hline $40-49$ & 6,920 & $2,754(39.8)$ & $2,701(39.0)$ & $1,157(16.7)$ & $308(4.5)$ & \\
\hline $50-59$ & 8,634 & $3,192(37.0)$ & $3,395(39 \cdot 3)$ & $1,636(18.9)$ & $411(4.8)$ & \\
\hline $60-69$ & 5,173 & $1,659(32.1)$ & $2,108(40.8)$ & $1,089(21.1)$ & $317(6.0)$ & \\
\hline$\geq 70$ & 1,343 & $364(27.1)$ & $574(42.7)$ & $309(23.0)$ & $96(7.2)$ & \\
\hline
\end{tabular}

Values are presented as number (\%).

${ }^{a}$ Derived from a linear-by-linear association test between sex and size.

roid nodules, clinical parameters included in the components of MetS showed differences according to the presence of thyroid nodule. Therefore, we performed the logistic regression analyses to identify independent associations between the presence of thyroid nodules and other clinical parameters including the components of MetS. Being female, older age, higher level of BMI, waist circumference, and SBP, lower HDL-C, and overt/ subclinical thyrotoxic state were independently associated with the presence of thyroid nodules (Table 4, model 1). In the analysis including the presence of MetS as an independent variable showed female, older age, higher BMI, MetS, and overt/subclinical thyrotoxic state were independently associated with the presence of thyroid nodules (Table 4, model 2). The number of components satisfying MetS criteria was also independently associated with the presence of thyroid nodules (Table 4, model 3 )

\section{DISCUSSION}

This study is the first multicenter-based health checkup study investigating the prevalence of thyroid nodules and their associating clinical parameters in Korea. In this study, the prevalence of thyroid nodules at health checkups was $34.0 \%$ (27.0\% in men, $41.7 \%$ in women), and various clinical factors, including sex, age, BMI, MetS, and thyroid function, were independent- ly associated with the presence of thyroid nodules. We distinguished thyroid pure cysts from thyroid nodules and excluded pure cysts in the nodular group because thyroid pure cysts have very low risk of malignancy and their clinical importance is different from thyroid solid nodules $[15,16]$. Our results showing similar prevalence of thyroid pure cysts in each group of age also suggest different pathophysiology between thyroid pure cysts and solid nodules. We also excluded pure cysts in the non-nodular group because we intended to compare the subjects without any focal lesion and those with thyroid nodules. Previous single-center studies in Korea have shown a wide range of thyroid nodule prevalence of $14.1 \%$ to $40.9 \%$ because of the inclusion of different study populations with varying sex and age compositions [5-8]. Studies performed in the United States, Europe, and China have also reported the prevalence ranged $19.0 \%$ to $46.6 \%$ for thyroid incidentalomas in screening with thyroid US [12,17-19]. A previous study of 8,307 subjects who underwent health checkups in a single health checkup center in Korea from January 2002 to December 2003 reported a prevalence of $41.0 \%$ for thyroid nodules and a higher prevalence in women (29.0\% in men, $42.2 \%$ in women) and in older age groups [6]. We found a similar prevalence of thyroid nodules as that reported by previous studies, but we found a more consistent results showing women's higher prevalence of thyroid nodules in every age group. We also demon- 
Table 3. Comparison of anthropometric and metabolic parameters according to the presence and size of thyroid nodules

\begin{tabular}{|c|c|c|c|}
\hline Variable & Non-nodular group $(n=38,502)$ & Nodular group $(n=24,757)$ & $p$ value \\
\hline$\overline{\text { Sex }}$ & & & $<0.001^{\mathrm{a}}$ \\
\hline Men & $22,622(58.8)$ & $9,919(40.1)$ & \\
\hline Women & $15,880(41.2)$ & $14,838(59.9)$ & \\
\hline Age, yr & $47.5 \pm 10.3$ & $52.7 \pm 10.4$ & $<0.001^{b}$ \\
\hline $\mathrm{BMI}^{\mathrm{c}}, \mathrm{kg} / \mathrm{m}^{2}$ & $23.4 \pm 3.1$ & $23.6 \pm 3.0$ & $<0.001^{b}$ \\
\hline$<25$ & $26,850(71.0)$ & $16,329(69.9)$ & $0.003^{\mathrm{a}}$ \\
\hline$\geq 25$ & $10,961(29.0)$ & $7,035(30.0)$ & $0.003^{\mathrm{a}}$ \\
\hline Waist circumference ${ }^{\mathrm{d}}, \mathrm{cm}$ & $84.0 \pm 8.4$ & $84.7 \pm 8.3$ & $<0.001^{b}$ \\
\hline$<90$ in men, $<85$ in women & $26,375(69 \cdot 7)$ & $14,444(61.8)$ & $<0.001^{\mathrm{a}}$ \\
\hline$\geq 90$ in men, $\geq 85$ in women & $11,464(30.3)$ & $8,913(38.2)$ & $<0.001^{\mathrm{a}}$ \\
\hline Body fat, \% & $24.5 \pm 5.6$ & $26.6 \pm 6.0$ & $<0.001^{b}$ \\
\hline Systolic blood pressure, mmHg & $116.6 \pm 15 \cdot 3$ & $118.6 \pm 16.3$ & $<0.001^{\mathrm{b}}$ \\
\hline Diastolic blood pressure, $\mathrm{mmHg}$ & $74.6 \pm 11.8$ & $74.8 \pm 11.7$ & $0.036^{\mathrm{b}}$ \\
\hline Fasting glucose, $\mathrm{mg} / \mathrm{dL}$ & $96.3 \pm 19.9$ & $97.0 \pm 20.1$ & $<0.001^{b}$ \\
\hline HbAic, \% & $5.7 \pm 0.7$ & $5.8 \pm 0.7$ & $<0.001^{b}$ \\
\hline AST, IU/L & $25.0 \pm 14.1$ & $24.7 \pm 13.7$ & $0.002^{b}$ \\
\hline ALT, IU/L & $27.0 \pm 20.9$ & $25.4 \pm 19.0$ & $<0.001^{b}$ \\
\hline GGT, IU/L & $36.2 \pm 41.4$ & $31.0 \pm 32.5$ & $<0.001^{b}$ \\
\hline BUN, mg/dL & $13.4 \pm 3.5$ & $13.8 \pm 3.7$ & $<0.001^{b}$ \\
\hline Creatinine, $\mathrm{mg} / \mathrm{dL}$ & $1.00 \pm 0.20$ & $0.96 \pm 0.21$ & $<0.001^{b}$ \\
\hline Uric acid, mg/dL & $5.6 \pm 1.5$ & $5.2 \pm 1.4$ & $<0.001^{b}$ \\
\hline Total cholesterol, mg/dL & $195 \cdot 9 \pm 34 \cdot 3$ & $198.6 \pm 34.9$ & $<0.001^{\mathrm{b}}$ \\
\hline Triglycerides, mg/dL & $115.9 \pm 76.2$ & $111.4 \pm 70.3$ & $<0.001^{b}$ \\
\hline HDL-C, mg/dL & $54.0 \pm 13.5$ & $54 \cdot 7 \pm 13 \cdot 4$ & $<0.001^{b}$ \\
\hline LDL-C, mg/dL & $114.1 \pm 30.7$ & $116.8 \pm 31.0$ & $<0.001^{b}$ \\
\hline MetS & & & $<0.001^{\mathrm{a}}$ \\
\hline No & $32,113(86.4)$ & $19,214(83.9)$ & \\
\hline Yes & $5,069(13.6)$ & $3,696(16.1)$ & \\
\hline No. of MetS components & & & $<0.001^{e}$ \\
\hline 0 & $14,342(38.6)$ & $7,518(32.8)$ & \\
\hline 1 & $10,794(29.0)$ & $6,822(29.8)$ & \\
\hline 2 & $6,977(18.8)$ & $4,874(21.3)$ & \\
\hline 3 & $3,633(9.8)$ & $2.546(11.1)$ & \\
\hline 4 & $1,259(3.4)$ & $983(4 \cdot 3)$ & \\
\hline 5 & $176(0.5)$ & $167(0.7)$ & \\
\hline Free $\mathrm{T}_{4}^{\mathrm{f}}$ & $1.2 \pm 0.3$ & $1.2 \pm 0.9$ & $0.926^{\mathrm{b}}$ \\
\hline TSF, median (interquartile range) f,g $^{\text {fo }}$ & $0.50(10.06)$ & $0.44(9.92)$ & $0.002^{b}$ \\
\hline Thyroid hormone state ${ }^{f}$ & & & $<0.001^{\mathrm{e}}$ \\
\hline Overt/subclinical hypothyroid state & $2,242(6.1)$ & $1,320(5.8)$ & \\
\hline Euthyroid state & $33,819(92.0)$ & $20,702(91.0)$ & \\
\hline Overt/subclinical thyrotoxic state & $689(1.9)$ & $718(3.2)$ & \\
\hline
\end{tabular}

Values are presented as number $(\%)$ or mean \pm SD unless otherwise indicated.

BMI, body mass index; HbA1c, glycosylated hemoglobin; AST, aspartate aminotransferase; ALT, alanine aminotransferase; GGT, $\gamma$-glutamyl transpeptidase; BUN, blood urea nitrogen; HDL-C, high density lipoprotein cholesterol; LDL-C, low density lipoprotein cholesterol; MetS, metabolic syndrome; T4, thyroxine; TSH, thyroid stimulating hormone.

${ }^{a}$ Derived from the chi-squared test.

${ }^{\mathrm{b}}$ Derived from Student $t$ test.

${ }^{\mathrm{c}}$ Data were available in 61,176 subjects.

${ }^{\mathrm{d}}$ Data were available in 61,197 subjects.

${ }^{\mathrm{e}}$ Derived from a linear-by-linear association test.

${ }^{\mathrm{f}}$ Data were available in 61,698 subjects.

${ }^{\mathrm{g}}$ Log-transformed variables were used in statistical analyses. 
Table 4. Clinical factors associating the presence of thyroid nodules

\begin{tabular}{|c|c|c|c|c|c|c|c|c|}
\hline \multirow[t]{2}{*}{ Variable } & \multicolumn{2}{|c|}{ Univariate analysis } & \multicolumn{2}{|c|}{$\begin{array}{c}\text { Multivariable } \\
\text { analysis model } 1^{\mathrm{a}}\end{array}$} & \multicolumn{2}{|c|}{$\begin{array}{c}\text { Multivariable } \\
\text { analysis model } 2^{\mathrm{b}}\end{array}$} & \multicolumn{2}{|c|}{$\begin{array}{c}\text { Multivariable } \\
\text { analysis model } 3^{\mathrm{c}}\end{array}$} \\
\hline & OR & $95 \% \mathrm{CI}$ & OR & $95 \%$ CI & OR & $95 \% \mathrm{CI}$ & OR & $95 \%$ CI \\
\hline \multicolumn{9}{|l|}{ Sex } \\
\hline Men & 1 & - & 1 & - & 1 & - & 1 & - \\
\hline Women & 1.929 & $1.870-1.991$ & 2.363 & $2.273-2.457$ & 2.294 & $2.212-2.379$ & 2.295 & $2.213-2.380$ \\
\hline Age, yr & 1.045 & $1.043-1.046$ & 1.046 & $1.044-1.048$ & 1.047 & $1.045^{-1.049}$ & 1.047 & $1.045^{-1.049}$ \\
\hline BMI, kg/m² & 1.020 & $1.015^{-1.025}$ & 1.017 & $1.006-1.029$ & 1.038 & $1.031-1.045$ & 1.033 & $1.026-1.041$ \\
\hline $\mathrm{HbAic}, \%$ & 1.162 & $1.136-1.188$ & 1.003 & $0.967-1.041$ & 0.997 & $0.971-1.024$ & 0.991 & $0.964-1.018$ \\
\hline Waist circumference, $\mathrm{cm}$ & 1.010 & $1.008-1.012$ & 1.007 & $1.003-1.012$ & - & - & - & - \\
\hline Systolic blood pressure, $\mathrm{mmHg}$ & 1.008 & $1.007-1.009$ & 1.002 & $1.001-1.004$ & - & - & - & - \\
\hline Triglycerides, mg/dL & 0.999 & $0.998-0.999$ & 0.999 & $0.999-1.000$ & - & - & - & - \\
\hline HDL-C, mg/dL & 1.003 & $1.002-1.004$ & 0.998 & $0.996-0.999$ & - & - & - & - \\
\hline Fasting glucose, mg/dL & 1.002 & $1.001-1.003$ & 0.9997 & $0.998-1.001$ & - & - & - & - \\
\hline \multicolumn{9}{|l|}{ MetS } \\
\hline No & 1 & - & - & - & 1 & - & - & - \\
\hline Yes & 1.221 & $1.168-1.276$ & - & - & 1.043 & $0.989-1.101$ & - & - \\
\hline \multicolumn{9}{|l|}{ No. of MetS components } \\
\hline o & 1 & - & - & - & - & - & - & - \\
\hline 1 & 1.207 & $1.160-1.256$ & - & - & - & - & 1.061 & $1.014-1.110$ \\
\hline 2 & 1.325 & $1.267-1.384$ & - & - & - & - & 1.076 & $1.018-1.135$ \\
\hline 3 & 1.339 & $1.266-1.415$ & - & - & - & - & 1.086 & $1.013-1.165$ \\
\hline 4 & 1.479 & $1.359-1.609$ & - & - & - & - & 1.140 & $1.031-1.262$ \\
\hline 5 & 1.869 & $1.522-2.294$ & - & - & - & - & 1.278 & $1.017-1.606$ \\
\hline \multicolumn{9}{|l|}{ Thyroid hormone state } \\
\hline $\begin{array}{l}\text { Overt/subclinical hypothyroid } \\
\text { state }\end{array}$ & 0.983 & $0.918-1.052$ & 0.789 & $0.734-0.849$ & 0.790 & $0.735-0.850$ & 0.789 & $0.734-0.849$ \\
\hline Euthyroid state & 1 & - & 1 & - & 1 & - & 1 & - \\
\hline $\begin{array}{l}\text { Overt/subclinical thyrotoxic } \\
\text { state }\end{array}$ & 1.688 & $1.526-1.866$ & 1.620 & $1.455^{-1.803}$ & 1.628 & $1.463-1.812$ & 1.629 & $1.464-1.814$ \\
\hline
\end{tabular}

The dependent variable was the presence of thyroid nodules in all statistical models. Clinical factors associating the presence of thyroid nodules entered as independent variables.

OR, odds ratio; CI, confidence interval; BMI, body mass index; HbAıc, glycosylated hemoglobin; HDL-C, high density lipoprotein cholesterol; MetS, metabolic syndrome.

${ }^{a}$ Sex, age, BMI, HbA1c, waist circumference, systolic blood pressure, fasting glucose, triglycerides, HDL-C, fasting glucose, and thyroid function were included as an independent variable in multivariable analysis model 1.

${ }^{\mathrm{b}} \mathrm{Sex}$, age, BMI, HbAic, MetS, and thyroid function were included as an independent variable in multivariable analysis model 2. ${ }^{\mathrm{c}} \mathrm{Sex}$, age, BMI, HbA1c, number of MetS components, and thyroid function were included as an independent variable in multivariable analysis model 3 .

strated a higher proportion of larger nodules in women and a continuing increase of the prevalence and size of thyroid nodules according to age. To our knowledge, this study includes the largest population (72,319 sub- jects) and is the first multicenter study to investigate the prevalence of thyroid nodules detected at health checkups in Korea. Therefore, our study demonstrates stronger sex and age differences in the prevalence and size of 
thyroid nodules than do previous studies. The thyroid nodules detected in asymptomatic healthy subjects are generally small and, as expected, we found that $76.1 \%$ of nodules measured $\leq 1.0 \mathrm{~cm}$ in maximal diameter. This size distribution is also consistent with that reported in previous studies $[6,20]$.

Some studies reported that the presence of thyroid nodules was associated with metabolic disturbances [1113,21,22]. In earlier studies, the prevalence of thyroid nodules was higher in subjects with the MetS and impaired glucose metabolism [11,21], and those with thyroid nodules had a higher prevalence of obesity, the metabolic syndrome, prediabetes, and diabetes $[12,13,22]$. We also observed an independent association between the presence of thyroid nodules and a higher BMI and MetS. Interestingly, the number of components satisfying MetS criteria was associated with the presence of thyroid nodules. One possible mechanism to explain the association between thyroid nodules and metabolic disturbances is a TSH-dependent mechanism including leptin signaling. Leptin directly and indirectly increases the secretion of thyrotropin-releasing hormone, which results in an increase of TSH secretion [23]. Serum leptin concentration increases in proportion to increasing fat mass, and insulin administration increases serum leptin levels $[24,25]$. Thus, it has been postulated that obesity and insulin resistance increase TSH secretion via leptin signaling, which results in thyroid volume expansion and nodule formation. However, in our data, TSH level was lower in the subjects with thyroid nodules compared with those without nodules, and the nodular group had a higher frequency of overt/subclinical thyrotoxic state and lower frequency of overt/subclinical hypothyroid state. One previous study found no association between the TSH level and thyroid nodule presence despite finding an association between TSH level and thyroid volume [11]. Thus, TSH-independent mechanisms might be involved in the association between thyroid nodule formation and metabolic disturbances.

Insulin-like growth factor 1 (IGF-1) promotes the progression of mitosis in many types of cells and results in cell proliferation, differentiation, and apoptosis [26]. This potent mitogen functions through its binding to the IGF-1 receptor (IGF-1R), and the signal transduction through IGF-1R leads to abnormal hyperplasia, differentiation, and apoptosis of thyrocytes [27-29]. A recent study reported that the expression of IGF-1 and IGF-1R was significantly higher in the tissues of follicular adenomas and nodular goiters compared with normal thyroid tissues [29]. It is well-known, meanwhile, that IGF-1 and IGF-1R share structural homology with insulin and the insulin receptor, respectively [30]. Taken together, hyperinsulinemia in obesity and the insulin-resistant status might induce thyroid nodule formation through the insulin IGF-1-signaling pathway. This insulin/IGF-1 pathway could be a TSH-independent mechanism that might explain the association between thyroid nodule formation and metabolic disturbances.

As mentioned above, overt/subclinical thyrotoxic state (lower TSH level) was associated with an increased chance of having thyroid nodules, and overt/subclinical hypothyroid state (higher TSH level) was associated with a decreased chance in the present study. Because TSH is a major regulator of the growth and differentiation of thyrocytes, the TSH level had been expected to be higher in subjects with thyroid nodules than in those without nodules. Although we cannot clearly explain our results contrary to expectations, there is a possibility that our data may include subjects who had already been diagnosed of thyroid nodule and maintained levothyroxine for TSH suppression at the time of health checkup because we did not exclude the subjects using any kind of medication, including thyroid hormone or antithyroid drugs. In addition, extremely high prevalence of "hot nodule" could result in this association. However, our data from over 72,000 cases consistently demonstrated the association between the higher prevalence of thyroid nodule and the lower TSH level after adjusting other clinical factors. Further studies to investigate the underlying mechanisms of this association are required.

This study highlights some important clinical implications. Our data showing a high prevalence of thyroid nodules in the subjects who underwent thyroid US at health checkups and increasing prevalence in older age groups indicate that the popularity of thyroid US has contributed to the increase in detection of thyroid nodules and cancer in the general population. This contribution of thyroid US is also supported by the observations that the nodule prevalence did not change with time in the subjects who underwent thyroid US and that the absolute number of people receiving thyroid US has increased every year from 4,804 cases in 2004 to 19,939 
cases in 2010. The result showing that most of the thyroid nodules detected at health checkups measured $\leq 1.0$ $\mathrm{cm}$ is also consistent with the contribution of thyroid US to the high prevalence of thyroid nodules. Nevertheless, our data confirm an association between thyroid nodule presence and metabolic disturbance, including obesity and MetS. Considering the increases in the prevalence of obesity, MetS, and diabetes in Korea, our data suggest that metabolic disturbances may play a role in the recent increase in the prevalence of thyroid nodules.

The limitations of this study include the cross-sectional study design. Although we adjusted for possible covariates, we could not confirm a causal link between the clinical parameters and thyroid nodule presence in this study. Investigating the risk factors for newly developed thyroid nodules in a prospective or retrospective cohort study may improve the strength of future studies. Another limitation is the lack of morphological analysis and further information about the thyroid nodules. We could not include the data of multiplicity, echogenicity, border irregularity, microcalcification, or spongi-form morphology of thyroid nodules because we extracted the data from free text of US reports using a computerized keyword extraction method. Investigation of the clinical parameters associated with thyroid nodules, such as suspicious morphology or cytologic findings, may have strong clinical impact. We found differences in several parameters, including the concentrations of AST, ALT, GGT, BUN, creatinine, and uric acid, between the nodular and non-nodular groups. However, the differences were not clinically significant, and we included some clinically applicable parameters in multivariable analyses.

In conclusion, we conducted a multicenter study to investigate the prevalence of thyroid nodules detected at health checkups and their associated clinical parameters. The high prevalence of thyroid nodules detected by thyroid US at health checkups suggests that increased detection of thyroid nodules has resulted in an increasing prevalence in the general population. However, the association between thyroid nodule presence and metabolic disturbances, including obesity and MetS, suggests that these metabolic disturbances may have also contributed to the increase in the prevalence of thyroid nodules in Korea.

\section{KEY MESSAGE}

1. The prevalence of thyroid nodules detected by thyroid ultrasound at health checkups was $34.2 \%$.

2. Thyroid nodules were more prevalent in women and older age groups.

3. Thyroid nodule presence was associated with sex, age, body mass index, and metabolic syndrome.

4. The association between thyroid nodule presence and metabolic disturbances suggests that metabolic disturbances may have also contributed to the increase in the prevalence of thyroid nodules in Korea.

\section{Conflict of interest}

No potential conflict of interest relevant to this article was reported.

\section{Acknowledgments}

This study was supported by National Evidence-based Healthcare Collaborating Agency, NECA (NA13-OO3) and by the Research Grant Number CB 2011-03-01 of Korean Foundation for Cancer Research.

\section{REFERENCES}

1. Mitchell J, Parangi S. The thyroid incidentaloma: an increasingly frequent consequence of radiologic imaging. Semin Ultrasound CT MR 2005;26:37-46.

2. Davies L, Ouellette M, Hunter M, Welch HG. The increasing incidence of small thyroid cancers: where are the cases coming from? Laryngoscope 2010;120:2446-2451.

3. Morris LG, Sikora AG, Tosteson TD, Davies L. The increasing incidence of thyroid cancer: the influence of access to care. Thyroid 2013;23:885-891.

4. Korea Centers for Disease Control and Prevention. Korea Health Statistics 2013: Korea National Health and Nutrition Examination Survey (KNHANES VI-1). Cheongju: Ministry of Health and Welfare, 2014.

5. Yim CH, Oh HJ, Chung HY, et al. Prevalence of thyroid nodules detected by ultrasonography in womens attending health check-ups. J Korean Soc Endocrinol 
2002;17:183-188.

6. Suk JH, Kim TY, Kim MK, et al. Prevalence of ultrasonographically-detected thyroid nodules in adults without previous history of thyroid disease. J Korean Endocr Soc 2006;21:389-393.

7. Kim JH, Park SJ, Kim SE, et al. Prevalence of thyroid nodules detected by ultrasonography in adult men attending health check-ups. J Korean Endocr Soc 2007;22:112-117.

8. Kim WJ, Kim JH, Park DW, et al. Prevalence of thyroid nodules detected by ultrasonography in adults for health check-ups and analysis of fine needle aspiration cytology. J Korean Endocr Soc 2008;23:413-419.

9. Knudsen N, Laurberg P, Perrild H, Bulow I, Ovesen L, Jorgensen T. Risk factors for goiter and thyroid nodules. Thyroid 2002;12:879-888.

10. Rezzonico J, Rezzonico M, Pusiol E, Pitoia F, Niepomniszcze $H$. Introducing the thyroid gland as another victim of the insulin resistance syndrome. Thyroid 2008;18:461-464.

11. Ayturk S, Gursoy A, Kut A, Anil C, Nar A, Tutuncu NB. Metabolic syndrome and its components are associated with increased thyroid volume and nodule prevalence in a mild-to-moderate iodine-deficient area. Eur J Endocrinol 2009;161:599-605.

12. Guo H, Sun M, He W, et al. The prevalence of thyroid nodules and its relationship with metabolic parameters in a Chinese community-based population aged over 40 years. Endocrine 2014;45:230-235.

13. Kim JY, Jung EJ, Park ST, et al. Body size and thyroid nodules in healthy Korean population. J Korean Surg Soc 2012;82:13-17.

14. Grundy SM, Cleeman JI, Daniels SR, et al. Diagnosis and management of the metabolic syndrome: an American Heart Association/National Heart, Lung, and Blood Institute Scientific Statement. Circulation 2005;112:2735-2752.

15. Horvath E, Majlis S, Rossi R, et al. An ultrasonogram reporting system for thyroid nodules stratifying cancer risk for clinical management. J Clin Endocrinol Metab 2009;94:1748-1751.

16. Brito JP, Gionfriddo MR, Al Nofal A, et al. The accuracy of thyroid nodule ultrasound to predict thyroid cancer: systematic review and meta-analysis. J Clin Endocrinol Metab 2014;99:1253-1263.

17. Brander A, Viikinkoski P, Nickels J, Kivisaari L. Thyroid gland: US screening in a random adult population. Ra- diology 1991;181:683-687.

18. Burguera B, Gharib H. Thyroid incidentalomas: prevalence, diagnosis, significance, and management. Endocrinol Metab Clin North Am 2000;29:187-203.

19. Volzke H, Ludemann J, Robinson DM, et al. The prevalence of undiagnosed thyroid disorders in a previously iodine-deficient area. Thyroid 2003;13:803-810.

20. Brander AE, Viikinkoski VP, Nickels JI, Kivisaari LM. Importance of thyroid abnormalities detected at US screening: a 5-year follow-up. Radiology 2000;215:801-806.

21. Anil C, Akkurt A, Ayturk S, Kut A, Gursoy A. Impaired glucose metabolism is a risk factor for increased thyroid volume and nodule prevalence in a mild-to-moderate iodine deficient area. Metabolism 2013;62:970-975.

22. Yin J, Wang C, Shao Q, et al. Relationship between the Prevalence of thyroid nodules and metabolic syndrome in the iodine-adequate area of Hangzhou, China: a cross-sectional and cohort study. Int J Endocrinol 2014;2014:675796.

23. Zimmermann-Belsing T, Brabant G, Holst JJ, Feldt-Rasmussen U. Circulating leptin and thyroid dysfunction. Eur J Endocrinol 2003;149:257-271.

24. Lewandowski K, Randeva HS, O'Callaghan CJ, et al. Effects of insulin and glucocorticoids on the leptin system are mediated through free leptin. Clin Endocrinol (Oxf) 2001;54:533-539.

25. Schmidt MI, Duncan BB, Vigo A, et al. Leptin and incident type 2 diabetes: risk or protection? Diabetologia 2006;49:2086-2096.

26. Jones JI, Clemmons DR. Insulin-like growth factors and their binding proteins: biological actions. Endocr Rev 1995;16:3-34.

27. Kimura T, Van Keymeulen A, Golstein J, Fusco A, Dumont JE, Roger PP. Regulation of thyroid cell proliferation by TSH and other factors: a critical evaluation of in vitro models. Endocr Rev 2001;22:631-656.

28. Hartog H, Wesseling J, Boezen HM, van der Graaf WT. The insulin-like growth factor 1 receptor in cancer: old focus, new future. Eur J Cancer 2007;43:1895-1904.

29. Liu YJ, Qiang W, Shi J, Lv SQ, Ji MJ, Shi BY. Expression and significance of IGF-1 and IGF-1R in thyroid nodules. Endocrine 2013;44:158-164.

30. Riedemann J, Macaulay VM. IGF1R signalling and its inhibition. Endocr Relat Cancer 2006;13 Suppl 1:S33-S43. 